INTERNATIONAL DESIGN CONFERENCE - DESIGN 2018

https://doi.org/10.21278/idc.2018.0161

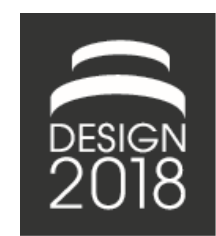

\title{
CROWDSOURCING IN PRODUCT DEVELOPMENT: CURRENT STATE AND FUTURE RESEARCH DIRECTIONS
}

\author{
H. L. Forbes and D. Schaefer
}

\begin{abstract}
The term crowdsourcing has been identified as a valuable paradigm in the open design movement. Despite its proven value, research focusing specifically on crowdsourcing in product development has been stagnating, with the general tone in this sector having moved from optimism to skepticism. In this paper, the authors present a literature analysis that reveals four hurdles limiting industry adoption. The authors are then able to suggest future research avenues that they anticipate will encourage adoption of crowdsourcing in professional product design and development settings in industry.
\end{abstract}

Keywords: crowdsourcing, product development, participatory design, collaborative design, open innovation

\section{Introduction}

In recent years, the application of open design models and principles has increased (Markman, 2016). As a consequence, many businesses have began to see the benefits of using the crowd as part of the product development process. A key paradigm of the open design movement is crowdsourcing. One of the most famous examples of crowdsourcing is Procter and Gamble's "Connect and Develop" which allows the organisation to "partner with the world's most innovative minds" by encouraging the crowd to submit product ideas and suggestions (Procter \& Gamble, 2017). As a consequence of "Connect and Develop", $45 \%$ of Procter and Gamble's key initiatives are now discovered externally and research and development "productivity has increased by 60\%" (Huston and Sakkab, 2006).

Despite initial momentum, however, the application of crowdsourcing in product development seems to be stagnating. As to be expected, the increased application of crowdsourcing has exposed aspects of the phenomena that, when not seriously considered, hinder the success of its application. As a consequence, the tone evoked by literature on crowdsourcing in product development has become increasingly negative. Papers such as "The value of crowdsourcing: can users really compete with professionals in generating new product ideas?" by Poetz et al. (2012) and "Identifying experts in the crowd for evaluation of engineering designs" by Burnap et al. (2017) suggest industry-wide skepticism towards crowdsourcing and the value of the crowd.

To understand the reason for this change in tone and the deceleration of crowdsourcing adoption, the authors performed an in-depth study of crowdsourcing literature in the context of product development. To guide this study, the following research questions were devised:

1. Does academic literature on crowdsourcing in product development signal any reason(s) for the decelerated adoption of crowdsourcing? If so, what are they?

2. How can these hurdles be overcome through future research? 
A total of 31 sources were analysed and synthesised, with the outcome being the four hurdles preventing wide spread adoption of crowdsourcing in product development. The hurdles include a lack of a clear definition, limited exploration of crowdsourcing implementation types, limited guidance on the effective application of crowdsourcing in product development and unbalanced exploration of crowdsourcing in all product development phases. For each hurdle, its existence is proven with existing literature, the reasoning for its existence is then considered before possible solutions and future research avenues are suggested. The aim of this paper is to provide the foundation for future research on this topic, with the ultimate outcome being an increase in the use of crowdsourcing in product development.

\section{Paper collection and selection}

The authors were interested in discovering how the academic perspective on this subject has evolved since its inception. The approach, therefore, was to find the earliest examples of literature on crowdsourcing in product development before collecting all papers from this point onwards. This allowed the authors to study the full spectrum of literature.

The following search phrases were used; "Crowdsourcing in Product Development", "Crowdsourcing in Design", "Crowdsourcing in Engineering Design" and "Crowdsourcing Product Design". These were inputted into the following literature databases; Google Scholar (scholar.google.com), Research Gate (researchgate.net), Scopus (www.scopus.com), Web of Science (webofknowledge.com) and Science Direct (sciencedirect.com). The following approach was then conducted:

1. "Crowdsourcing in Product Development" was inputted into Google Scholar

2. The earliest example of a paper on this subject was determined by filtering the results by year

3. The title and abstract of this paper was read and a decision was made on whether this paper was considered relevant to the subject according to the following criteria:

a. The source must be written in or translated to English

b. The source must not be self-published and must be associated with an official academic publication

c. The source must discuss the role of crowdsourcing within the context of the search phrase and not just mention the term

d. The source must discuss the role of crowdsourcing within the context of the search phrase and not just crowdsourcing in general

4. If the abstract met the criteria, it was listed as the earliest example of literature for this database

5. This process was then repeated for all databases and for all search phrases to find the earliest paper on crowdsourcing in the context of product development

6. Filters were then set to the determined time period on each database, each search phrase was used and papers were added to the collection based on the criteria

7. For every paper, the "related papers" shown on both Research Gate and Google Scholar were also considered for collection

8. The final step was scanning and selecting further papers from the references of the collected papers

Conducting this approach yielded a total of 45 sources. This selection was then refined by reading the full paper to determine whether the source definitely met the criteria listed above. This refinement process yielded a total of 31 papers. These were analysed and synthesised to reveal four hurdles for the adoption and use of crowdsourcing in product development.

\section{Critical literature review and discussion}

This section defines the four hurdles limiting the use of crowdsourcing in product development. Each hurdle is first highlighted with existing research, according to Research Question 1. Reasoning for the existence of this hurdle and solutions for overcoming the hurdle are then considered according to Research Question 2.

\subsection{The definition of crowdsourcing}

The first hurdle is a lack of clarity on the definition of crowdsourcing. Practitioners may therefore recognise a need to increase their understanding of the term, but current literature does not satisfy this need. Without true understanding of the term, effective application is unlikely. 
Firstly, academics cause a lack of clarity on the definition of crowdsourcing by combining or confusing crowdsourcing with other terms. For example, terms such as co-creation, collective intelligence and open source are often presented as synonymous with crowdsourcing and used interchangeably. Ramanujan et al. (2015) uses the term crowdsourcing and "cloud-based co-creation" synonymously while Zheng and Jakiela (2011) states that "one type of crowdsourcing is co-creation". Maher (2011) states that "crowdsourcing is part of a larger phenomenon of collective intelligence" while several other academics suggest that crowdsourcing is an example or demonstration of collective intelligence (Zheng et al., 2009). The open source movement is also often described as crowdsourcing but Howard et al. (2012) states that crowdsourcing is one of the four paradigms of the "new revolution of design" which includes "Open Innovation, Open Source, Crowdsourcing and Open Design". Howard et al. (2012) therefore, present crowdsourcing and open source as two distinct phenomena, while Olson and Rosacker (2013) state that "open source is crowdsourcing applied to software". It is clear that there is no agreed consensus on the terminology describing and associated with crowdsourcing and this understandably causes confusion over the definition (Gerth, 2012).

Further confusion regarding the definition of crowdsourcing has been exacerbated by categorisation of the phenomena. For example, Howe (2006) defines crowdsourcing using the perspective of "The Professional", "The Packager", "The Tinkerer" and "The Masses". Pan and Blevis (2011) on the other hand, defines crowdsourcing as having three "dimensions" which are "role-orientated crowdsourcing", "behaviour-orientated crowdsourcing" and "media-orientated crowdsourcing" and H. L. Zheng et al. (2011) state that "one type of crowdsourcing is co-creation". Recognising the multifaceted nature of the term is important in increasing the understanding of crowdsourcing but categorisation using different variables, as is demonstrated in the literature selection, has caused further confusion.

A solution to this hurdle is a standardised definiton that encompasses all forms of crowdsourcing. Furthermore, instead of ambiguously defining crowdsourcing categories as "types", academics should endeavour to be more specific. For example, Howe's (2011) categorisation of crowdsourcing should be defined as categorisation according to the role of the crowdsourcer. Should future publications ensure this granularity when categorising crowdsourcing, general understanding of the term can be improved.

\subsection{Limited exploration of crowdsourcing implementation types}

The second hurdle is the limited exploration of crowdsourcing implementation types. The authors define the term "implementation type" as the way in which an organisation performs crowdsourcing. For example, crowdsourcing for information via an open call to the public is one example of an "implementation type". This section first demonstrates the minimal representation of implementation types in current literature and then follows with a discussion on potential research avenues.

\subsubsection{Current categorisation of crowdsourcing}

Table 1 presents every source in the literature collection that includes categorisation of crowdsourcing.

Table 1. The categorisation of crowdsourcing in current literature

\begin{tabular}{|l|c|r|}
\hline Categorisation Variable & Reference(s) & Examples of Categories \\
\hline Organisational Purpose & $\begin{array}{c}\text { Erickson et al. (2012), } \\
\text { Erickson (2013) }\end{array}$ & $\begin{array}{r}\text { Productivity, Innovation, Knowledge } \\
\text { Capture, Marketing/Branding }\end{array}$ \\
\hline Perspective & $\begin{array}{c}\text { Howe (2006), } \\
\text { Pand Blevis (2011) }\end{array}$ & $\begin{array}{r}\text { Role-orientated, behaviour-orientated } \\
\text { and media-orientated crowdsourcing }\end{array}$ \\
\hline Implementation Type & $\begin{array}{c}\text { Gerth et al. (2012), } \\
\text { Panchal (2015) }\end{array}$ & $\begin{array}{r}\text { Crowdsourcing contests, open call with } \\
\text { direct rewards, open call with indirect } \\
\text { benefits, micro tasks }\end{array}$ \\
\hline Product Development Phase & $\begin{array}{c}\text { Maher (2011), } \\
\text { Preece et al. (2016), } \\
\text { Tran and Park (2012), } \\
\text { Wu et al. (2013) }\end{array}$ & $\begin{array}{r}\text { Crowdsourcing for planning, concept } \\
\text { development, system level design, detail } \\
\text { design }\end{array}$ \\
\hline
\end{tabular}


Of the 31 collected sources, only 10 considered crowdsourcing categories and only 2 considered crowdsourcing implementation types. The limited exploration of implementation types is a hurdle for crowdsourcing adoption because this form of crowdsourcing categorisation offers useful guidance on the application of crowdsourcing. By presenting all implementation types with their varying characteristics, organisations can select and apply based on their desired outcomes and circumstances. As a consequence, the process of applying crowdsourcing to product development is streamlined.

To overcome this hurdle, a standardised set of crowdsourcing implementation types should be presented. This solution, however, requires extensive research to ensure all facets of these implementation types are considered. For example, how a crowdsourcing campaign is implemented depends on a myriad of factors such as the size of the organisation, the complexity of the problem and the resources available to the organisation. Each of these factors need to be identified and then how these factors impact the implementation of crowdsourcing needs to be considered.

\subsubsection{Future research avenues for implementation types}

This section identifies possible research avenues on the subject of crowdsourcing implementation types. Firstly, the authors present evidence from current literature that suggests that the degree to which contributors communicate impacts crowdsourcing implementation. Howe (2008) states that crowdsourcing offers useful solutions to problems because contributors are "independent" and "dislocated" meaning they are not influenced by each other and are able to draw completely on their unique perspective. He states that by aggregating the ideas from these "independent" contributors, you are able to find a solution that "outperforms traditional ideation techniques" (Howe, 2008). To counter this idea, however, Nickerson et al. (2011) explores "how human interactions can be setup to encourage creativity in crowdsourcing activities". They suggest that crowdsourcing should be a collaborative process with "three generations" of contributors building and refining on the previous generation's ideas. Furthermore, Maher (2011) presents an idea for a crowdsourcing activity setup called "Crowd vs. Crowd" where crowdsourcers are arranged in teams to present ideas. Both of these academics provide evidence for the positive influence collaboration can have on crowdsourcing. As a consequence, the authors encourage future researchers to consider how varying degrees of crowd interaction influence the implementation of crowdsourcing.

Secondly, future research should include an investigation into how the involvement of the "expert" or the "professional" can influence crowdsourcing implementation. The literature that brought this issue to the attention of the authors is focused on evaluating crowdsourced design ideas. For example, Burnap et al. (2017) suggest that the current use of crowdsourcing for the evaluation of design ideas is flawed because "non-expert ideas overwhelm the crowd". They consider an "expert" to be a contributor that possesses "a high level of education". Howe (2008), however, suggested that the success of crowdsourcing is in the opportunity to tap into a diverse set of perspectives and that "a randomly selected collection of problem solvers outperforms a collection of the best individual problem solvers" (Howe, 2008). If it is assumed that "the best individual problem solvers" are what Burnap et al. (2017) regard as experts, then a method that uses only "expert" ideas is actively minimising the diversity of their sample. As a consequence, contributors from the crowd that are curated based on their level of education are unlikely to offer significant benefits beyond an internal R\&D team. There may, however, be an opportunity to involve the expert in some capacity that ensures both diverse and feasible ideas. Future research on implementation types should include an investigation into how increasing or decreasing the role of the expert influences the outcome of a crowdsourcing campaign.

Another consideration is whether a set of crowdsourcing implementation types should accommodate organisations that aren't willing to negotiate on their idea of a "high-quality solution". Burnap et al. (2017) highlight the issue by stating that all ideas should be compared to "the true objective". Brambilla (2015) also hint at a similar idea by judging the success of a crowdsourcing campaign on the number of "correct" and "precise" responses. The authors question how organisations can expect to find a novel solution if the quality of crowdsourced ideas are judged according to an existing solution. Furthermore, this existing solution is likely to have been created by an in-house team which means the available solutions are once again impacted by a biased view of expertise. The authors support Howe's (2008) theory on the aspects of crowdsourcing that encourage its success and therefore feel that methods that 
minimise diversity will not encourage valuable crowdsourcing. Perhaps organisations need to recognise the power of the crowd and sacrifice long held ideas of expertise and quality in order to apply crowdsourcing successfully. On the other hand, it is clear from current literature that receiving an excess of unsuitable crowdsourced solutions is considered a challenge. It is also likely that this is leading to slow adoption of crowdsourcing in engineering design. It is suggested, therefore, that offering a crowdsourcing implementation type that considers "an ideal solution" may be required in the short term to advance the adoption of crowdsourcing in product development. Future researchers may consider this in how the crowdsourcing brief is presented or by controlling the type of crowd involved in the implementation of crowdsourcing.

The consideration of contributor interaction, the role of the expert and comparison to the ideal solution in crowdsourcing should be included in future research on implementation types. By recognising these ideas, crowdsourcing implementation types should allow practitioners to overcome challenges and embrace the opportunities that crowdsourcing offers.

\subsection{Limited guidance on the application of crowdsourcing in product development}

The third hurdle is a lack of literature available on how to apply crowdsourcing in product development. This section demonstrates that as well as limited exploration of implementation types, general advice for the implementation of crowdsourcing in product development, is limited. Literature that does offer advice can be arranged in four areas. These are academic advice on the construction of crowdsourcing platforms, research on motivation for crowd participation, advice for structuring a crowdsourcing campaign brief and crowdsourcing frameworks to guide the application of crowdsourcing in product development.

Grace et al. (2015), Preece et al. (2016), Nebeling et al. (2012) and Jakiela (2009) suggest that advertising and collecting crowd contributions on a well-constructed platform is the key to effective crowdsourcing. Of the academics whose research lies in this area, the majority agree that the optimal crowdsourcing platform should itself be crowdsourced and customizable (Nebeling et al., 2012). Grace et al. (2015) suggest that in order to serve and encourage contributors, a platform should be created that is "iteratively designed by the users". Nebeling et al. (2012) similarly champion a crowdsourcing platform that is "componentized" and "customizable by user data and user suggestions" (Nebeling et al., 2012). Qin et al. (2016) state that in order for crowdsourcing to be adopted by organisations, the crowdsourcing platform must have the following characteristics:

- "User centred-focus" that ensures the platform is built with a primary focus on user needs

- "Iterative and modular" to ensure web development of the platform is reactive and can quickly be altered

- "Highly integrated with social media" to ensure contributors can receive adequate credit and support (Qin et al., 2016)

Preece et al. (2016) created and launched an improved crowdsourcing platform called "NatureNet" that followed these principles. Crowd contributions, with the improved platform, were shown to increase. These academics therefore unite in proving that a customizable crowdsourcing platform encourages increased involvement, by both contributors and organisations. A concern, however, is the limited consideration of other aspects of a crowdsourcing platform. For example, user customisation is found to be important but other important aspects such as user experience design and user interface design are hardly considered in current literature. Should a practitioner look to create a crowdsourcing platform from scratch, the guidance offered by current literature is limited.

The next area of literature is on contributor motivation. By understanding why contributors choose to become involved in crowdsourcing, organisations can launch campaigns that are more popular and therefore more effective. Unfortunately, of the 31 collected sources, only 2 discussed crowd motivations. Zheng et al. (2011) find that contributors may not be "universally driven by financial reward" but state "gaining recognition" was consistently a key motivator. Jakiela (2009) supports this conclusion by suggesting that "credit attribution" to contributors was important. While this conclusion is beneficial, there is no research to suggest how an organisation can maximise credit attribution. Furthermore, current literature is not specific to a crowdsourcing implementation type and this limits its 
value. For example, motivation for involvement in an open source project may differ from motivation for involvement in an open call crowdsourcing campaign. Motivations to be involved may also vary according to the gender of the contributor, the age of the contributor, the industry of the contributor and more. Significant research is therefore required to be able to offer valuable guidance to practitioners regarding contributor motivation.

Regarding the crowdsourcing campaign brief, Zheng et al. (2011) state that it must have "clearly defined requirements" and Alonso (2013) states the need for "clear instructions". Zheng et al. (2011) also state that the task presented must "allow autonomy" but contributors must "be confident they can find a solution". Wu et al. (2013), however, suggest that when using crowdsourcing for the evaluation of ideas, participants that are not familiar with the original project brief can evaluate designs most effectively (Wu et al., 2013). Zheng et al. (2011) and Alonso (2013) therefore comment on how the brief is written while $\mathrm{Wu}$ et al. (2013) consider how the brief should be distributed. This literature area is lacking in advice for application. For example, how should requirements be defined, how can a brief be devised to ensure "autonomy" for the crowd and how can the brief be presented clearly. If the crowd should be detached from the project, how can the brief be distributed to ensure the crowd is not influenced. These questions, need to be answered through future research to ensure advice on crowdsourcing in product development is genuinely applicable.

The final sector is on crowdsourcing frameworks that guide the application of crowdsourcing in product development. Panchal (2015) presents a framework consisting of three steps; "choosing a crowdsourcing initiative", "making design decisions" and "incentive design". He illustrates the application of this framework using game theory and also demonstrates how it can be integrated into an overarching product development framework. Saldanha et al. (2014) make a similar attempt to model the process of crowdsourcing, however, they do so by applying an existing ideation strategy. As is shown in Figure 1, Saldanha et al. (2014) suggest that contributions should be gradually refined by the crowd in a stage-gate filtering process. This is similar to Nickerson et al.'s (2011) "generational" crowdsourcing process as discussed previously.

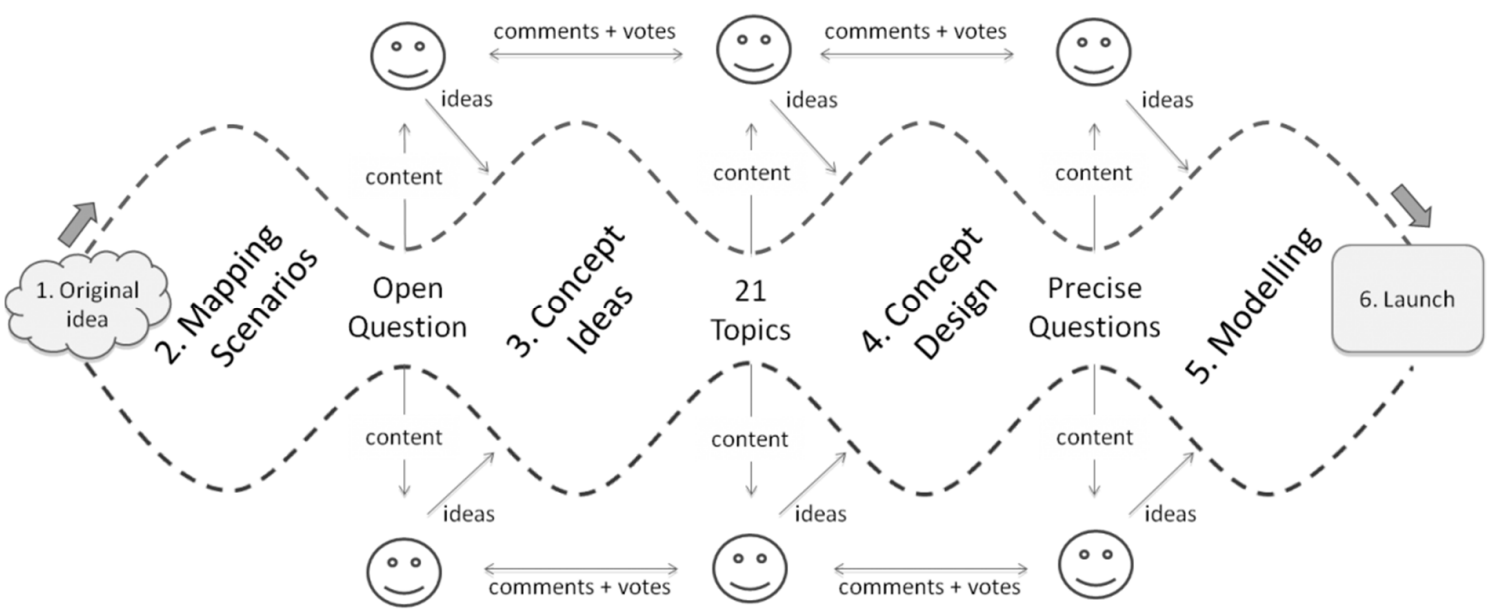

Figure 1. Saldanha et al.'s (2014) stage-gate process for the application of crowdsourcing in product development

The third example of a crowdsourcing framework, is that of Park et al. (2013) which allows companies to maintain a level of control over the ideas they receive. Park et al. (2013) presents a crowdsourcing framework that organises the crowd in teams with "expert design leads". Solutions are suggested by each team but a solution is only escalated after it has had approval from the design lead. This framework allows organisations to seek solutions from numerous and varied perspectives but an expected level of quality is known and managed throughout. These three examples each offer valuable advice, from diverse research, for crowdsourcing in product development. The shortcomings of this literature sector, however, is again its limited offering with only 3 academics involved in this research. Furthermore, only Panchal (2015) considers how the application of crowdsourcing may vary according to the organisations 
circumstances. Future research should have the aim to produce crowdsourcing frameworks that can be tailored according to the user's product development needs. This should encourage further adoption of crowdsourcing by offering greater support for a wider range of organisations.

By amalgamating all guidance on crowdsourcing in product development offered by academic sources, the authors in this section were able to identify key literature gaps and offer ideas for future research. In the next section, the literature selection is examined according to the phases of product development.

\subsection{Unbalanced exploration of crowdsourcing in the product development phases}

The vast majority of current literature considers crowdsourcing to be an activity solely for the ideation and concept evaluation stages of product development. Saldanha et al. (2014) only considers a framework for ideation, Nickerson et al. (2011) discuss creativity within crowdsourcing but only as part of ideation and Wu et al. (2013) and Xu et al. (2011) only discuss crowdsourcing for concept evaluation. Ren et al. (2013) states that "crowdsourcing can be used for ideation and evaluation", thus dismissing other aspects of product development. A trend among current literature has therefore emerged, as demonstrated in Figure 2, which suggests that crowdsourcing should be used predominantly in ideation and evaluation. While crowdsourcing may not ultimately be suitable for other aspects, the lack of research on other product development phases is concerning.

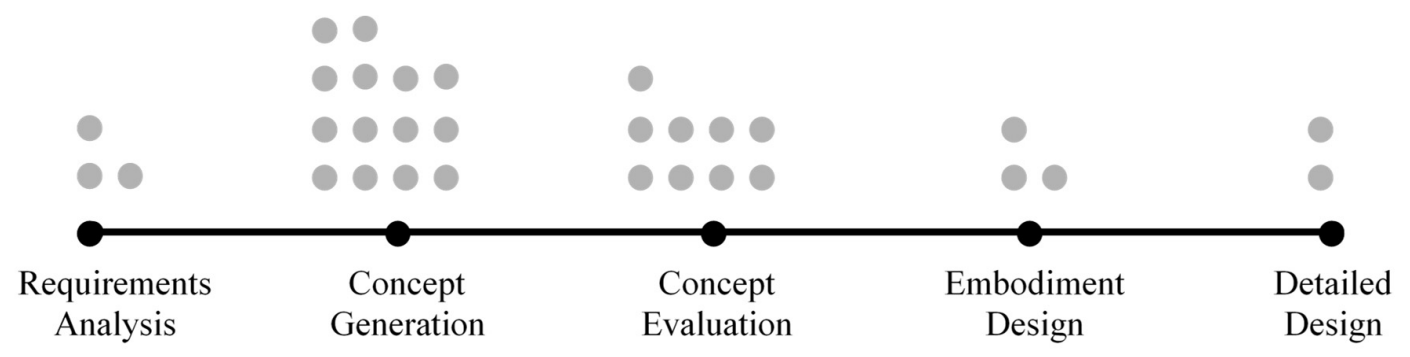

Figure 2. Current literature's exploration of crowdsourcing in each product development phase; One grey dot represents one source

There are two academics that seem to recognise the potential of crowdsourcing beyond ideation and evaluation, however. Panchal (2015), after presenting his framework, recognised that it should be extended in "different dimensions for the different design phases". Furthermore, Tran and Park (2012) investigated crowdsourcing in every aspect of the product development process. While neither of these papers offer detailed guidance on using crowdsourcing throughout the product development process they suggest that there is potential in this concept. The authors believe that by expanding on this area of research and determining whether crowdsourcing could be used throughout product development, new applications that offer new benefits could be discovered.

\section{Conclusions}

This paper shows the result of an analysis and synthesis of a selection of 31 papers on crowdsourcing in product development. The authors aim was to reveal hurdles in current literature that could offer an explanation to the limited adoption of crowdsourcing in product development. Four hurdles were identified which included a lack of a clear definition of crowdsourcing, limited exploration of crowdsourcing implementation types, limited guidance on the effective application of crowdsourcing in product development and unbalanced exploration of crowdsourcing in all parts of the product development process.

To fulfil the first research question, each hurdle was identified and evidence for their existence was presented. Regarding the definition of crowdsourcing, academics often used other terminology interchangeably and synonymously. Furthermore, other academics defined crowdsourcing by categorising the term into "types" and inconsistent categorisations led to further confusion. In order to fulfil the second research question, solutions for the hurdles were presented. Regarding the first hurdle, a standardised definition for crowdsourcing should be presented that incorporates all forms of 
crowdsourcing. Furthermore, academics should specify how they are categorising crowdsourcing, to avoid further confusion.

The second recognised hurdle led to an introduction of the term "implementation type" which is the way in which an organisation performs crowdsourcing. By presenting all implementation types with their varying characteristics, organisations can select and apply based on their desired outcomes and circumstances. Of all the selected sources only two considered the actual implementation of crowdsourcing, providing evidence for a lack of research in this area. The solution to this hurdle, with reference to the second research question, is to present a set of implementation types that considers all the ways in which a crowdsourcing campaign could be influenced or altered.

The third hurdle was the limited research on effectively applying crowdsourcing in product development. Literature gaps were identified by splitting this area of research into four areas; academic advice on the construction of crowdsourcing platforms, research on motivation for crowd participation, advice for structuring a crowdsourcing campaign brief and crowdsourcing frameworks to guide the application of crowdsourcing in product development. The fourth hurdle was the limited research on crowdsourcing in all phases of the product development process. Figure 2 illustrated the strong bias towards considering crowdsourcing for ideation and concept evaluation. In the case of the third and fourth hurdle, the second research question was addressed by identifying the literature gaps and offering avenues for further research for each research area.

Beyond the specific research questions, the authors also presented further considerations regarding crowdsourcing implementation types for product development. It was found that an investigation into the different levels of communication between the crowd is required as well as considering how concepts of "the expert" and "the ideal solution" can be incorporated into a set of implementation types.

Overall, the authors believe that addressing the hurdles presented in this paper could lead to an acceleration in the use of crowdsourcing in product development. By clearly defining crowdsourcing, it is likely to be better understood. By categorising crowdsourcing into implementation types and offering more guidance on the use of crowdsourcing, organisations can more easily apply it. By investigating crowdsourcing in all design phases, further applications of crowdsourcing can emerge and organisations can find new ways to reap the rewards of crowdsourcing and the open design movement.

\section{References}

Alonso, O. (2013), "Implementing crowdsourcing-based relevance experimentation: an industrial perspective", Information retrieval, Vol. 16 No. 2, pp. 101-120. https://doi.org/10.1007/s10791-012-9204-1

Brambilla, M., Ceri, S., Mauri, A. and Volonterio, R. (2015), “An Explorative Approach for Crowdsourcing Tasks Design.”, 24th International Conference on World Wide Web, Florence, Italy, May 18-22, 2015, New York, NY, USA pp. 75-90. https://doi.org/10.1145/2740908.2743972

Burnap, A., Gerth, R., Gonzalez, R. and Papalambros, P.Y. (2017), "Identifying experts in the crowd for evaluation of engineering designs", The Journal of Engineering Design, Vol. 28 No. 5, pp. 317-337. https://doi.org/10.1080/09544828.2017.1316013

Erickson, L.B. (2013), Hanging with the right crowd: Crowdsourcing as a new business practice for innovation, productivity, knowledge capture, and marketing. [online] Penn State University. Available at: https://etda.libraries.psu.edu/catalog/17524 (accessed 21.12.2017).

Erickson, L.B., Petrick, I. and Trauth, E.M. (2012), "Organizational uses of the crowd: developing a framework for the study of crowdsourcing." Proceedings of the SIGMIS-CPR 2012 / 50th Annual Conference on Computers and People Research, Milwaukee, Wisconsin, USA, May 31 - June 02, 2012, New York, NY, USA, pp. 155-158. https://doi.org/10.1145/2214091.2214133

Gerth, R.J., Burnap, A. and Papalambros, P. (2012), Crowdsourcing: A primer and its implications for systems engineering. [online] Defense Technical Information Centre. Available at: http://www.dtic.mil/docs/citations/ADA566807 (accessed 17.12.2017).

Grace, K., Maher, M.L., Preece, J., Yeh, T., Stangle, A. and Boston, C. (2015), "A process model for crowdsourcing design: A case study in citizen science", Design Computing and Cognition '14, Springer, Cham, pp. 245-262. https://doi.org/10.1007/978-3-319-14956-1_14

Howard, T.J., Achiche, S., Özkil, A. and McAloone, T.C. (2012), "Open design and crowdsourcing: maturity, methodology and business models." Proceedings of DESIGN 2012 / the 12th International Design Conference, Dubrovnik, Croatia, May 21 - 24, 2012, pp. 155-158.

Howe, J. (2006), “The rise of crowdsourcing”, Wired, No. 14, pp. 1-4. 
Howe, J. (2008), Crowdsourcing: How the Power of The Crowd is Driving the Future of Business, Crown Publishing Group New York, NY, USA.

Huston, L. and Sakkab, N. (2006), Connect and Develop: Inside P\&G's new model for innovation, Harvard Business Review, Vol. 84 No. 3, pp. 58-66.

Jakiela, M.J. (2009), Contribution Attribution as the possible next step for crowdsourced engineering design and product development. [online] Open Scholarship. Available at: https://openscholarship.wustl.edu/cgi/viewcontent.cgi?referer=https://scholar.google.co.uk/\&httpsredir=1\&ar ticle $=1105 \&$ context $=$ law_journal_law_(accessed 17.12.2017).

Maher, M. (2011), "Design Creativity Research: From the Individual to the Crowd", In: Taura T., Nagai Y. (Ed.), Design Creativity, Springer, London, UK, pp. 303-317. https://doi.org/10.1007/978-0-85729-224-7_7

Markman, A.B. (2016), Open Innovation: Academic and Practical Perspectives on the Journey from Idea to Market, Oxford University Press, Oxford, UK

Nebeling, M., Leone, S. and Norrie, M. (2012), “Crowdsourced web engineering and design”, Proceedings of Web Engineering 2012 / 12th International Conference of Web Engineering, Berlin, Germany, July 22 - 26, 2012, Springer, Berlin, pp. 31-45.

Nickerson, J.V., Sakamoto, Y. and Yu, L. (2011), "Structures for creativity: The crowdsourcing of design", Proceedings of Human factors in Computing Systems 2011 / Annual Conference Extended Abstracts on Human Factors in computing systems, May 8, 2011, ACM, New York, NY, USA, pp. 1-4.

Olson, D.L. and Rosacker, K. (2013), "Crowdsourcing and open source software participation", Service Business, Vol. 7 No. 4, pp. 499-511. https://doi.org/10.1007/s11628-012-0176-4

Pan, Y. and Blevis, E. (2011), "A survey of crowdsourcing as a means of collaboration and the implications of crowdsourcing for interaction design", Proceedings of CTS 2011 / International Conference on Collaboration Technologies, May 23 - 27, 2011, Philadelphia, PA, USA, IEEE, pp. 397-403. https://doi.org/10.1109/CTS.2011.5928716

Panchal, J.H. (2015), "Using Crowds in Engineering Design: Towards a Holistic Framework", Proceedings of ICED 2015 / 20th International Conference on Engineering Design, Milan, Italy, July 27 - 30, 2011, The Design Society, pp. 27-30.

Park, C.H., Son, K., Lee, J.H. and Bae, S.H. (2013), "Crowd vs. crowd: large-scale cooperative design through open team competition", Proceedings of The Conference on Computer Supported Cooperative Work / 16th International Conference on Computer Supported Cooperative Work, San Antonio, Texas, February 23 - 27, 2013, ACM, pp. 1275-1284.

Poetz, M.K. and Schreier, M. (2012), "The value of crowdsourcing: can users really compete with professionals in generating new product ideas?”, Journal of Product Innovation Management, Vol. 29 No. 2, pp. 245-256. https://doi.org/10.1111/j.1540-5885.2011.00893.x

Preece, J., Boston, C., Maher, M.L., Grace, K. and Yeh, T. (2016), "From Crowdsourcing Technology Design to Participatory Design and Back Again!”, Proceedings of The European Conference on Social Media 2016 / 3rd European Conference on Social Media, Caen, France, 12-13 July, 2016, Media Research, pp. 315-323.

Procter \& Gamble (2017), What is Connect \& Develop?. [online] Procter \& Gamble. Available at: www.pgconnectdevelop.com/what-is-connect-develop (accessed 11.01.2017).

Qin, S., van Der Velde, D., Chatzakis, E., McStea, T. and Smith, N. (2016), "Exploring barriers and opportunities in adopting crowdsourcing based new product development in manufacturing SMEs", Chinese Journal of Mechanical Engineering, Vol. 29 No. 6, pp. 1052-1066. https://doi.org/10.3901/CJME.2016.0808.089

Ramanujan, D., Vinayak, Y.N., Reid, T. and Ramani, K. (2015), "Informing early design via crowd-based cocreation", Proceedings of IDETC-CIEC 2015 / International Design engineering Technical Conferences and Computers and Information in Engineering Conference, Boston, Massachusetts, USA, August 2-5, 2015, Boston, Massachusetts, ASME, pp. V007T06A043. https://doi.org/10.1115/DETC2015-47826

Ren, Y., Burnap, A. and Papalambros, P. (2013), "Quantification of perceptual design attributes using a crowd", Proceedings of ICED 2013 /19th International Conference on Engineering Design, Seoul, South Korea, 19-22 August, USA, pp. 139-148.

Saldanha, F.P., Cohendet, P. and Pozzebon, M. (2014), "Challenging the Stage-Gate Model in Crowdsourcing: The Case of Fiat Mio in Brazil", Technology Innovation Management Review, Vol. 4 No. 9, pp. 28-35.

Tran, A. and Park, J. (2012), "Crowd participation pattern in the phases of a product development process that utilizes crowdsourcing”, Industrial Engineering and Management Systems, Vol. 11 No. 3, pp. 266-275. https://doi.org/10.7232/iems.2012.11.3.266

Wu, W., Luther, K., Pavel, A., Hartmann, B., Dow, S. and Agrawala, M. (2013), CrowdCritter: strategies for crowdsourcing visual design critique. [online] University of California at Berkeley. Available at: www2.eecs.berkeley.edu/Pubs/TechRpts/2013/EECS-2013-95 (accessed 12.01.2017). 
Xu, A. and Bailey, B.P. (2011), "A crowdsourcing model for receiving design critique", Proceedings of Human factors in Computing Systems 2011 / Annual Conference Extended Abstracts on Human Factors in computing systems, May 8, 2011, ACM, New York, NY, USA, pp. 1183-1188.

Zheng, H., Li, D. and Hou, W. (2011), "Task design, motivation, and participation in crowdsourcing contests", International Journal of Electronic Commerce, Vol. 15 No. 4, pp. 57-88. https://doi.org/10.2753/JEC10864415150402

Zheng, J. and Jakiela, M.J. (2009), "An investigation of the productivity difference in mechanical embodiment design between face-to-face and threaded online collaboration", Proceedings of IDETC-CIEC 2009 / International Design Engineering Technical Conferences and Computers and Information in Engineering Conference, San Diego, California, USA, August 30 - September 2, 2009, ASME, pp. 1173-1182. https://doi.org/10.1115/DETC2009-87070

Hannah Louise Forbes, PhD Candidate

The University of Liverpool, Civil Engineering and Industrial Design

8 Creffield Road, W5 3HP Ealing, United Kingdom

Email: hannah.forbes@liverpool.ac.uk 